\title{
Positive attributes in children and reduced risk of future psychopathology
}

Pablo Vidal-Ribas, Robert Goodman and Argyris Stringaris

\section{Background}

There is little research on children's positive attributes and their association with psychiatric outcomes.

\section{Aims \\ To examine the hypothesis that children's positive attributes are associated with a reduced risk of developing psychopathology in future.}

\section{Method}

Positive attributes, measured with the Youth Strengths Inventory (YSI) and psychiatric outcomes were assessed on two occasions over 3 years in a large epidemiological sample of British children and adolescents $(n=5325)$.

\section{Results}

The YSI showed high to moderate cross-informant correlations and longitudinal stability. Children scoring high on positive attributes at baseline had fewer psychiatric symptoms and disorders at follow-up, adjusting for symptoms at baseline, disorder at baseline and child and family factors. Analyses with propensity score matching also suggested that positive attributes decrease the likelihood of psychiatric morbidity.

\section{Conclusions}

Children's positive attributes are associated with significantly less psychopathology across time and may be a target for intervention.

\section{Declaration of interest}

None.

\section{Copyright and usage}

(c) The Royal College of Psychiatrists 2015. This is an open access article distributed under the terms of the Creative commons Attribution (CC BY) licence.
It has been suggested that children with positive attributes, such as being affectionate, responsible or generous, may be protected from developing psychopathology. ${ }^{1-3}$ If this is true, facilitating the emergence of positive attributes - in addition to, or instead of attempts to reduce psychiatric symptoms - may be a particularly attractive way to promote well-being in youth. ${ }^{4,5}$ However, the evidence-base for such an assertion is still weak in several respects. First, there is little research about the positive attributes of children and adolescents from the general population and no longitudinal studies starting in childhood. ${ }^{1,3}$ Second, there is little research into whether positive attributes are merely the flipside of the absence of psychiatric symptoms, with reflectiveness for instance, being a positive attribute that is more or less synonymous with the absence of a psychiatric symptom, namely impulsiveness; yet it is crucial to demonstrate that positive attributes have predictive value across time even when adjusting for psychopathology. Third, most previous research failed to control for factors - including social or family characteristics that are plausible confounders of the relationship between positive attributes and psychiatric outcomes.

Our aim with this study was to address these shortcomings and test the importance of positive attributes by using data from The 2004 British Child and Adolescent Mental Health Survey (B-CAMHS04), ${ }^{6,7}$ a longitudinal multi-informant study of young people in the general population. To date, few studies have employed parent-reported measures of positive attributes. ${ }^{5,8}$ Therefore, we first established how well parents' ratings of children's positive attributes can be measured in the general population employing the Youth Strengths Inventory (YSI) from the Development and Well-Being Assessment (DAWBA), ${ }^{9,10}$ by analysing its psychometric properties and the interrater correlations. Second, using path analysis we examined whether children's positive attributes are a construct sufficiently distinct from parental accounts of children's mental illness. Third, we tested the hypothesis that the report of higher levels of positive attributes at baseline reduces the risk of psychopathology over a 3 -year period. We measured outcome using dimensional measures of psychopathology, psychiatric diagnoses as well as psychosocial adjustment indices. Finally, we used propensity scores ${ }^{1-13}$ to match young people on baseline psychopathology and plausible confounders - to estimate the potential treatment effects that positive attributes might have on the risk for future psychopathology. Propensity score matching is a process that attempts to emulate a randomised controlled trial (RCT). Thus, to reduce selection bias we matched groups with high and low positive attributes on observed covariates, such as psychopathology, family variables and socioeconomic status.

\section{Method}

\section{Participants}

The B-CAMHS04 involved a sample of $5-16$ year olds $(n=7977)$ representative of the general British population; it has previously been described in detail. ${ }^{6}$ The study used 'child benefit' (a state benefit payable at that time in Great Britain for each child in a family) to develop a sampling frame of 5- to 16-year-olds in different postal sectors in England, Wales and Scotland. After excluding families with no recorded postal code, it was estimated that this represented $90 \%$ of all British children. Out of the 12294 contacted, there were $n=1085$ who opted out and $n=713$ who were non-eligible or had moved without trace, leaving 10496 who were approached in person. Of those, $n=7977$ participated ( $65 \%$ of those selected; $76 \%$ of those approached). If the child was aged 5-10, a face-to-face interview was conducted with the parent and if the child was aged 11-16, the parent was interviewed first followed by the young person. In 2007, i.e. 36 months after the baseline survey, ${ }^{7}$ families were approached once more unless they had previously opted out or the child was known to have died. Of the original $n=7977$ participants, $n=5325$ (67\%) participated in the detailed follow-up. ${ }^{7}$ All study procedures received multicentre research ethics committee approval and informed consent was obtained from parents and assent from children participants. 


\section{Assessment}

\section{Development and Well-Being Assessment}

The DAWBA ${ }^{9,10}$ is a structured interview administered by lay interviewers who also record verbatim accounts of problems. The questions are closely related to DSM-IV and ICD-10 14,15 diagnostic criteria and focus on current problems. The kappa ( $\kappa)$ statistic for chance-corrected agreement between two raters was 0.86 for any disorder (s.e.=0.04), 0.57 for internalising disorders (s.e. $=0.11$ ) and 0.98 for externalising disorders $($ s.e. $=0.02) .{ }^{9}$ Values of $\kappa<0$ indicate no agreement, $0-0.20$ slight agreement, $0.21-0.40$ fair agreement, $0.41-0.60$ moderate agreement, $0.61-0.80$ substantial agreement and $0.81-1$ almost perfect agreement. ${ }^{16}$ Children were assigned a diagnosis only if their symptoms were causing significant distress or social impairment The DAWBA was completed at baseline and at 36 months (further information on the DAWBA is available from http://www.dawba.info). This paper focuses on the overall presence of disorder (i.e. any DSM-IV disorder), externalising disorders (the combination of conduct, oppositional defiant and attention-deficit hyperactivity disorders) and internalising disorders (the combination of depressive and anxiety disorders).

\section{Youth Strengths Inventory}

The YSI forms part of the DAWBA (Section N) and asks about positive attributes of the child, with similar but not identical parent-report and youth-report versions. The first part of the YSI asks how applicable various descriptions are to the child (for example generous, affectionate, caring), while the second part asks about things the child does that please the parents (for parent report) or that the child is proud of (for self-report). Examples include being good with friends, helpful at home and polite. The parent version has 12 items in each part, whereas the self-report version has 8 items in the first part and 11 items in the second part. Each item is scored on a three-point Likert scale (i.e., no, 0; a little,1; a lot, 2), with scores ranging from 0 to 48 for the parent version and 0 to 37 for the self-report version. Examining the psychometric properties, we found that all YSI scales showed high internal consistencies, similar to other existing strengths measures. ${ }^{4,17,18}$ For parent report, Cronbach's alphas were 0.84 at baseline (mean 39.2, s.d. $=6.0$, median $40, \mathrm{IQR}=36-44$ ) and 0.86 at 36 months (mean 38.9, s.d. $=6.4$, median $40, \mathrm{IQR}=36-44$ ). For child report, they were 0.77 at baseline (mean 27.1, s.d. $=5.1$, median 27, $\mathrm{IQR}=24-31$ ) and 0.73 at follow-up (mean 28.3, s.d. $=3.7$, median $28, \mathrm{IQR}=26-31$ ). A preliminary factor analysis yielded to an unrotated single-factor structure for all informants and time points. For the parent-reported YSI scores, this single factor explained $79 \%$ of the variance at both time points. For the youth-reported YSI scores, the variance explained by this single factor was $83 \%$ in 2004 and $81 \%$ in 2007 . It should be noted that the item 'polite' was dropped from the factor analysis of youthreported positive attributes in 2007 because of zero variance (i.e. all children answered 'A lot' in this item). Parent-rated YSI scores were used in the analyses unless otherwise specified.

\section{Strengths and Difficulties Questionnaire}

The Strengths and Difficulties Questionnaire (SDQ) asks about 25 attributes, some positive and others negative; respondents use a three-point Likert scale to indicate how far each attribute applies to the target child. ${ }^{19}$ The 25 items are divided between five scales of five items each, generating scores for emotional symptoms, conduct problems, hyperactivity-inattention, peer problems and prosocial behaviour; all but the last are summed to generate a total difficulties score ranging $0-40$. The SDQ also has an impact supplement ${ }^{19}$ that asks whether the respondent thinks that the child or youth has a problem, and if so, enquires further about overall distress, social impairment, burden and chronicity. This instrument has robust psychometric properties. ${ }^{20,21}$ In our sample, Cronbach's alphas for parent reports were 0.84 at baseline (mean 5.9, s.d. $=4.8$ ) and 0.86 at 36 months (mean 5.7, s.d. $=4.6$ ). It is important to note that in this study, the SDQ was used to generate a difficulties score, but not to generate a strengths score: the strengths score used in the analyses reported in this paper was based on the YSI. The SDQ was used in addition to the DAWBA because there is increasing recognition of the great importance of assessing psychopathology as a dimension in addition to the diagnostic approach. ${ }^{22}$

\section{Psychosocial adjustment}

Measures of psychosocial adjustment such as contact with psychiatric services, self-harm, truancy and contact with police were gathered in the baseline survey (2004). A participant was coded as having ever experienced one of these outcomes as rated by youth, teacher or parent report. In the follow-up survey (2007), informants were asked again about the same outcomes - we analysed new onsets of these outcomes.

\section{Family factors}

Sociodemographic details were collected at the parental interview covering the following information (a) ethnic group, (b) housing tenure (rented accommodation $v$. owner-occupiers), (c) gross household income, (d) maternal highest educational qualification, (e) family type (reconstituted with step-parent and/or step/halfsiblings $v$. other, lone-parent $v$. other), (f) parental anxiety and depression, assessed with the 12-item version of the General Health Questionnaire, ${ }^{23}$ (g) family discord, assessed with the general functioning scale of the McMaster Family Assessment Device, which includes 12 items, scored on a 1-4 scale with a maximum score of $48,{ }^{24}$ and (h) stressful life events during the child's lifetime, including parental separation, court appearance, bereavement and serious illness or accident. ${ }^{25}$

\section{Child factors}

Parents also provided information about (a) age, (b) gender, (c) their child's general health, using a five-point Likert scale, including the report of any physical disorders affecting their child, (d) neurodevelopmental disorders like cerebral palsy, difficulties with coordination, epilepsy and muscle disease or weakness and (e) 'generalised learning disability' (intellectual disability, referred to as learning disability in UK health services). Parents and teachers were asked to estimate each child's mental age, and teachers reported whether a child had a written statement of special educational needs related to cognitive and intellectual needs (including specific, moderate, severe and profound intellectual difficulties, but not distinguishing between them). For the purpose of these analyses, a child was considered to have a 'generalised learning disability' when one or both informants estimated that mental age was $60 \%$ or less of the chronological age (such as a mental age of 6 or less at a chronological age of 10).

\section{Statistical analyses}

\section{Interrater associations and longitudinal stability}

We examined whether there was a correspondence between parent-rated positive attributes and their children's self-perceptions. Concurrent and longitudinal associations of positive attributes 
scores were tested using Pearson's correlations within and across informants.

\section{Distinction between positive attributes and psychiatric symptoms across time}

A path analysis model was estimated to test whether total SDQ score (symptoms) and YSI score (positive attributes) exhibited distinguishable predictions across time.

\section{Positive attributes predicting future psychiatric symptoms}

The prediction of psychiatric symptoms by positive attributes was estimated in regression models with the total SDQ symptom score as the outcome (i.e. at 36 month) and baseline YSI score (positive attributes) as the predictor. In subsequent steps, regression models were adjusted for total SDQ symptom score at baseline, as well as relevant family factors (i.e. ethnicity, single-parent family, reconstituted family, maternal highest educational qualification, gross household income, housing tenure, family functioning, General Health Questionnaire and life events) and child factors (i.e. age, gender, general health, neurodevelopmental disorder, generalised learning disability and any psychiatric disorder at baseline) as covariates. ${ }^{1,8,26-28}$ This way we were able to test the specific association between positive attributes and psychiatric outcomes without the overestimation bias because of common related factors.

\section{Positive attributes predicting future psychiatric disorders}

The prediction of psychiatric diagnoses at 36-month follow-up (dependent variable) by baseline positive attributes was estimated in logistic regression models where baseline YSI score was used as an independent variable. We predicted three domains of psychiatric disorders; these were 'any psychiatric disorder', 'emotionalinternalising disorder', and 'disruptive behaviour-externalising disorder'. In adjusted models, diagnoses at baseline were used as covariates. In subsequent steps, the same child and family factors employed as covariates in the prediction of psychiatric symptoms were also added to these models. In addition, to examine whether parental ratings of positive attributes may lead to psychiatric disorders or be a consequence of them, we employed path analytical models. Doing so, we were able to look at the longitudinal association taking into account the correlation at baseline and follow-up.

\section{Association with psychosocial adjustment}

We employed logistic regression models in which the independent variable was positive attributes at baseline, and the dependent variables were the new emergence of the following factors in the 36-month follow-up: contact with psychiatric services, self-harm, truancy and contact with police. The models were examined unadjusted and adjusted for baseline psychiatric symptoms.

\section{Difference in symptoms at follow-up between matched groups differing in level of positive attributes}

Propensity score matching is an attempt to reduce bias in causal inference in observational studies. Through this method, a 'treated' group (for example high positive attributes) is matched on plausible confounders to a 'control' group (for example low positive attributes). If a good balance is achieved (i.e. approximately equal distribution of baseline covariates in both groups) and assuming that this model includes all relevant confounders, then this observational study should emulate what a RCT does by randomisation. Hence, a difference in the outcome (for example symptoms at follow-up) can be attributed to being 'treated' or not - unless there were strong confounders that were neither measured directly nor correlated with matching variables that were measured.

Propensity score analysis has several advantages over multiple linear regression approaches. First, when a good covariate balance is achieved, propensity score analysis does not rely on the correct specification of the functional form of the relationship (for example linearity or log linearity) between the outcome and the covariates, which is not the case with linear regression models when covariate distributions are very different between groups. ${ }^{29,30}$ Second, propensity score methods make it easier to determine whether the model has been correctly specified than with regression approaches. ${ }^{11}$ Finally, propensity score analyses are considered objective in the sense that the model is specified without relying on the outcome. (It is also possible to combine propensity score analyses with regression adjustment to reduce slight imbalances in the covariates and increase precision. $)^{31-33}$

In this study we used a propensity score matching approach to test whether children only differing in levels of positive attributes at baseline showed different levels of psychiatric symptoms at follow-up. ${ }^{11}$ Our aim was to compare children at both extremes of the distribution of levels of positive attributes. A binary variable of baseline positive attributes was computed selecting the extremes of the distribution of this variable (high positive attributes $v$. low positive attributes) using percentiles 20 and 80 as cut-off points. Doing so, we kept approximately the same proportion of people in each group. We matched both groups on the same covariates that were adjusted for in the previous regression models, plus psychiatric symptoms and disorder at baseline. In this case, a logit regression model was used to estimate the propensity score. The resulting propensity score was the predicted probability of belonging to the high positive attributes group for each child. Propensity score matching was performed employing a one-toone nearest-neighbour method within a caliper (or distance) of 0.5 . $^{11,34}$ With one-to-one nearest-neighbour matching, only one 'control' participant is selected for each 'treated' participant, namely the one whose propensity score is closest to the 'treated' participant. ${ }^{12}$ Caliper is defined as the difference in propensity scores between selected matches. By setting a caliper of 0.5 we pre-specify the largest allowable absolute difference in propensity scores for matched participants, thus ensuring closer balance. We only analysed observations that were inside the common support area. That is, there might be substantial overlap of the propensity score distributions in the two groups, but potentially density differences. Therefore, we discard individuals with propensity score values outside the range of the other group, thereby also ensuring a better balance. ${ }^{12}$ Balance of the covariates was assessed before and after matching using a measure of standardised bias. ${ }^{13}$ Standardised differences of means $<0.20$ are acceptable, ${ }^{12,13}$ and differences $<0.10$ are considered negligible ${ }^{35}$ (i.e. no mean differences in a covariate between groups). Regression models were employed to test the difference in means of SDQ total score at follow-up between matched groups as well as the change of SDQ total score over time in each group. Cohen's $d$ were calculated as a measure of effect size.

The Stata 11 software package for Windows was employed to test all study hypotheses, except for the path analyses, which were run with MPlus version $7 .^{36}$

\section{Results}

\section{Interrater correlation and longitudinal stability}

As shown in Table 1, there was a moderately strong cross-sectional correlation between parent- and child-rated positive attributes as measured with the YSI. The stability of positive attributes scores within informants ranged from moderate in children to high in 


\begin{tabular}{|c|c|c|c|}
\hline \multirow[b]{3}{*}{ Positive attributes score } & \multicolumn{3}{|c|}{ Positive attributes score, $r(95 \% \mathrm{Cl}) n$} \\
\hline & \multicolumn{2}{|c|}{ Baseline } & \multirow{2}{*}{$\begin{array}{c}36 \text { months } \\
\text { Parent }\end{array}$} \\
\hline & Parent & Child & \\
\hline \multicolumn{4}{|l|}{ Baseline } \\
\hline Parent & - & - & - \\
\hline Child & $\mathbf{0 . 3 0}(0.24-0.36) 838$ & - & - \\
\hline \multicolumn{4}{|l|}{36 months } \\
\hline Parent & $\mathbf{0 . 6 4}(0.62-0.65) 4921$ & $0.24(0.18-0.31) 838$ & - \\
\hline Child & $\mathbf{0 . 2 9}(0.23-0.35) 838$ & $\mathbf{0 . 4 6}(0.41-0.51) 923$ & $\mathbf{0 . 3 0}(0.24-0.36) 838$ \\
\hline
\end{tabular}

parents. Finally, positive attributes scores at baseline were significantly better (as evidenced in non-overlapping CIs) in predicting positive attributes scores at 36 months within informants than across informants.

\section{Distinction between positive attributes and symptoms across time}

The path analysis model in Fig. 1 shows that the within-domain prediction is much stronger than the across-domain prediction: positive attributes are a better predictor of positive attributes, whereas symptoms are a better predictor of symptoms (as evidenced in non-overlapping CIs).

\section{Positive attributes predicting future psychiatric symptoms}

As seen in Table 2, higher levels of positive attributes measured at baseline were significantly predictive of fewer psychiatric symptoms at follow-up. This was even true in models adjusted for SDQ total symptom score at baseline, family factors and child factors.

\section{Positive attributes predicting future psychiatric disorders}

Table 3 shows that a higher level of parent-rated positive attributes at baseline was a significant predictor of less psychiatric disorders at follow-up, no matter what domain of disorder was predicted. This was even true when adjusting for baseline disorder, family factors and child factors.

Figure 2 shows that positive attributes predicted less psychiatric disorders (beta coefficient range: -0.19 to -0.32 ) to a significantly higher extent than disorders predicted less positive attributes (beta coefficient range: -0.05 to -0.08 ).

\section{Association with psychosocial adjustment}

As can be seen in Table 4, parent-rated positive attributes were significant predictors of less subsequent psychiatric and police Baseline

Follow-up

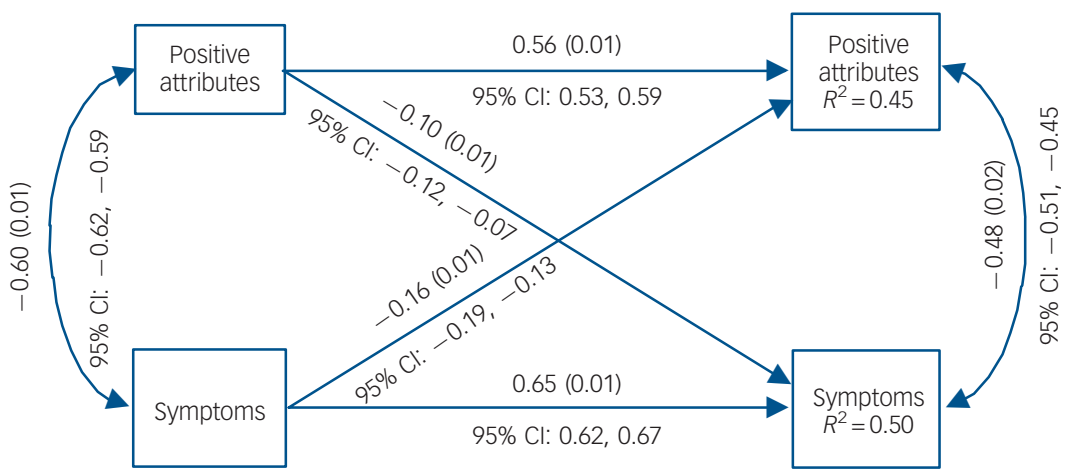

Fig. 1 Path analysis of the relationship between positive attributes and Strengths and Difficulties Questionnaire (SDQ) total difficulties (symptoms) score across time.

Significant paths $(P<0.05)$ and correlations with standard errors and $95 \%$ confidence intervals are presented as straight and curved lines respectively. $R^{2}$, proportion of variance explained.

Table 2 Association between positive attributes score at baseline and Strengths and Difficulties Questionnaire (SDQ) total difficulties (symptoms) score at follow-up in adjusted and unadjusted models ${ }^{\text {a }}$

Outcome: SDQ score at 36-month follow-up predicted by: $\beta(95 \% \mathrm{Cl}) R^{2}$

Positive attributes score only $\quad-\mathbf{0 . 4 8}(-0.51$ to -0.46$) 0.22$

Positive attributes score adjusted for baseline SDQ difficulties score $-\mathbf{0 . 1 0}(-0.12$ to -0.07$) 0.47$

Positive attributes score adjusted for baseline SDQ difficulties score and family factors $-\mathbf{0 . 1 0}(-0.12$ to -0.07$) 0.47$

Positive attributes score adjusted for baseline SDQ difficulties score and child factors $-0.09(-0.12$ to -0.07$) 0.48$

$\beta$, standardised regression coefficient; $R^{2}$, proportion of variance

a. Family factors are ethnicity, single parent family, reconstituted family, maternal highest education, gross household income, housing tenure, family functioning, General Health Questionnaire and life events. Child factors are age, gender, general health, neurodevelopmental disorder, generalised learning disability and any psychiatric disorder at baseline. All findings in bold are significant $(P<0.05)$. 
Any disorder predicted by:

Positive attributes only

Positive attributes adjusted for any disorder at baseline

$0.42(0.39-0.46)$

Positive attributes adjusted for any disorder at baseline and family factors

$\mathbf{0 . 5 7}(0.51-0.63)$

Positive attributes adjusted for any disorder at baseline and child factors

$0.59(0.53-0.66)$

Any emotional disorder predicted by:

Positive attributes only

$0.60(0.53-0.66)$

Positive attributes adjusted for any emotional disorder at baseline

$\mathbf{0 . 6 2}(0.56-0.70)$

$0.69(0.61-0.78)$

Positive attributes adjusted for any emotional disorder at baseline and family factors

$0.74(0.64-0.85)$

Positive attributes adjusted for any emotional disorder at baseline and child factors

$0.73(0.64-0.83)$

Any externalising disorder predicted by:

Positive attributes only

Positive attributes adjusted for any externalising disorder at baseline

$0.40(0.36-0.45)$

Positive attributes adjusted for any externalising disorder at baseline and family factors

Positive attributes adjusted for any externalising disorder at baseline and child factors

$\mathbf{0 . 5 4}(0.48-0.62)$

$0.61(0.52-0.70)$

$0.55(0.48-0.63)$

a. Family factors are ethnicity, single parent family, reconstituted family, maternal highest education, gross household income, housing tenure, family functioning, General Health Questionnaire and life events. Child factors are age, gender, general health, neurodevelopmental disorder and generalised learning disability. All findings in bold are significant $(P<0.05)$. Note that odds ratios significantly less than 1 represent a protective effect.

Baseline

(a)

(b)

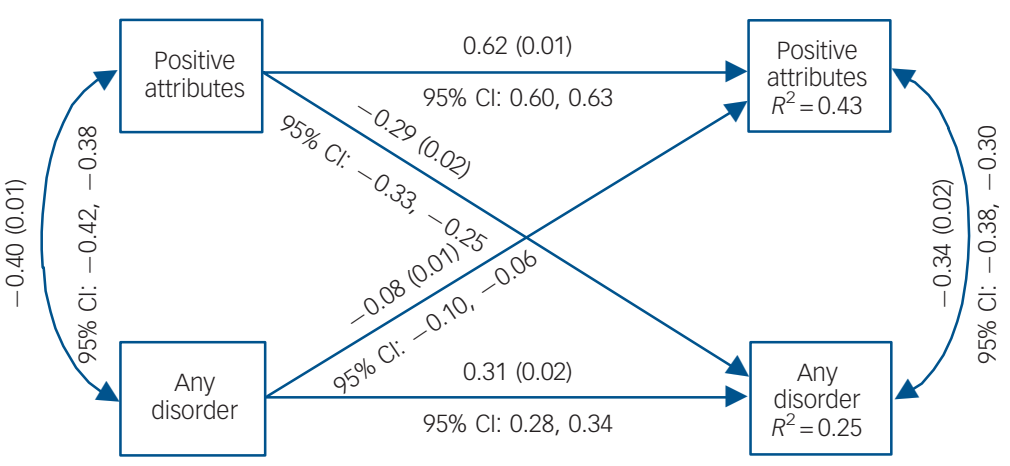

(c)
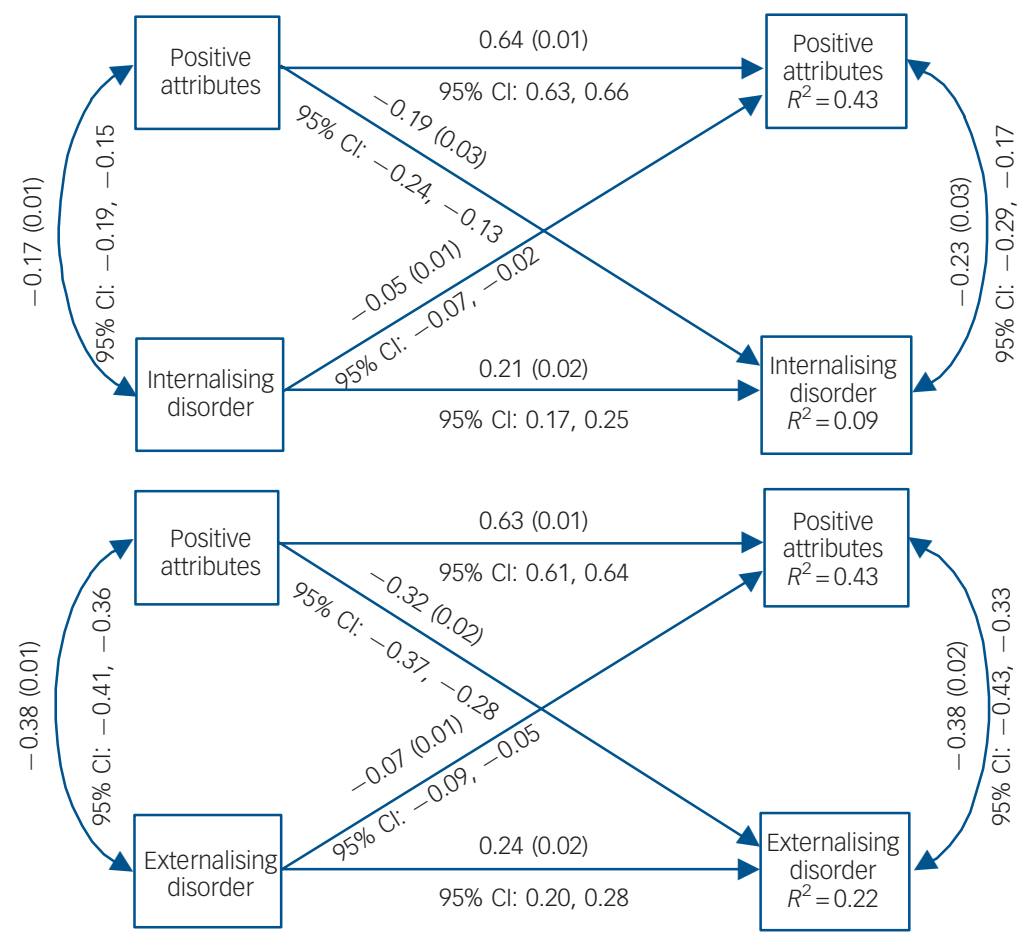

Fig. 2 Path analyses of the relation between positive attributes and any domain of disorder (a), any internalising disorder (b) and any externalising disorder (c) across time.

Significant paths $(P<0.001)$ and correlations with standard errors and $95 \%$ confidence intervals are presented as straight and curved lines respectively. $R^{2}$, proportion of variance explained. 
Table 4 Standardised positive attributes at baseline as predictors of a new onset of psychiatric contact, self- harm, truancy and police contact at follow-up, with and without adjustment for baseline symptoms ${ }^{a}$

Association of positive attributes at baseline with outcome at 36 months, OR (95\% CI) Unadjusted for baseline symptoms Adjusted for baseline symptoms

\begin{tabular}{|c|c|c|}
\hline Predicting new psychiatric contact & $\mathbf{0 . 6 0}(0.53-0.69)$ & $0.83(0.70-0.99)$ \\
\hline Predicting new self harm & $\mathbf{0 . 7 0}(0.63-0.79)$ & $0.87(0.75-1.01)$ \\
\hline Predicting new truancy & $\mathbf{0 . 5 8}(0.49-0.70)$ & $0.81(0.63-1.04)$ \\
\hline Predicting new police contact & $\mathbf{0 . 6 4}(0.58-0.71)$ & $0.75(0.66-0.86)$ \\
\hline
\end{tabular}

Odds ratios with confidence intervals are presented from logistic regression models with each of the outcomes as dependent variables and positive attributes as an independent variable either unadjusted or adjusted for baseline total Strengths and Difficulties Questionnaire score. All findings in bold are significant $(P<0.05)$. Note that odds ratios significantly less than 1 represent a protective effect.

contact in individuals who had not had such contact previously, even with adjustment for baseline psychiatric symptoms. By contrast, unadjusted prediction to new truancy or self-harm did not remain significant once adjusted for baseline psychiatric symptoms.

\section{Difference in symptoms at follow-up between matched groups differing in level of positive attributes}

As seen in Fig. 3, the reduction in standardised bias was acceptable after carrying out the propensity score matching; there was only one covariate with a standardised bias $>0.10$. The mean standardised bias in the covariates was $42.3 \%$ (s.d. $=39.5$, range 9.2-162.3) before matching, and decreased to $3.7 \%$ (s.d. $=2.8$, range $0.3-10.5$ ) after matching. The outcome analyses (after matching) revealed that there were significant differences in the SDQ total score at follow-up between matched groups with high and low levels of positive attributes (effect size $0.25, \quad 95 \%$ CI $0.11-0.38$, $P<0.001)$. Moreover, the change in SDQ total score over time was significant for the matched low positive attribute group (effect size 0.39 ; $95 \%$ CI $0.08-0.69, P<0.05)$ but not for the matched high positive attribute group (effect size $0.15,95 \% \mathrm{CI}-0.18$ to 0.47, $P=0.372$ ) (Fig. 4).

\section{Discussion}

\section{Main findings}

This study examined the extent to which parental perceptions of positive attributes in their own children predicted psychiatric outcomes. Analyses were carried out in a large epidemiological sample of young children and adolescents employing a longitudinal design. Overall, we found that higher levels of parental perceptions of positive attributes added to the prediction of fewer future

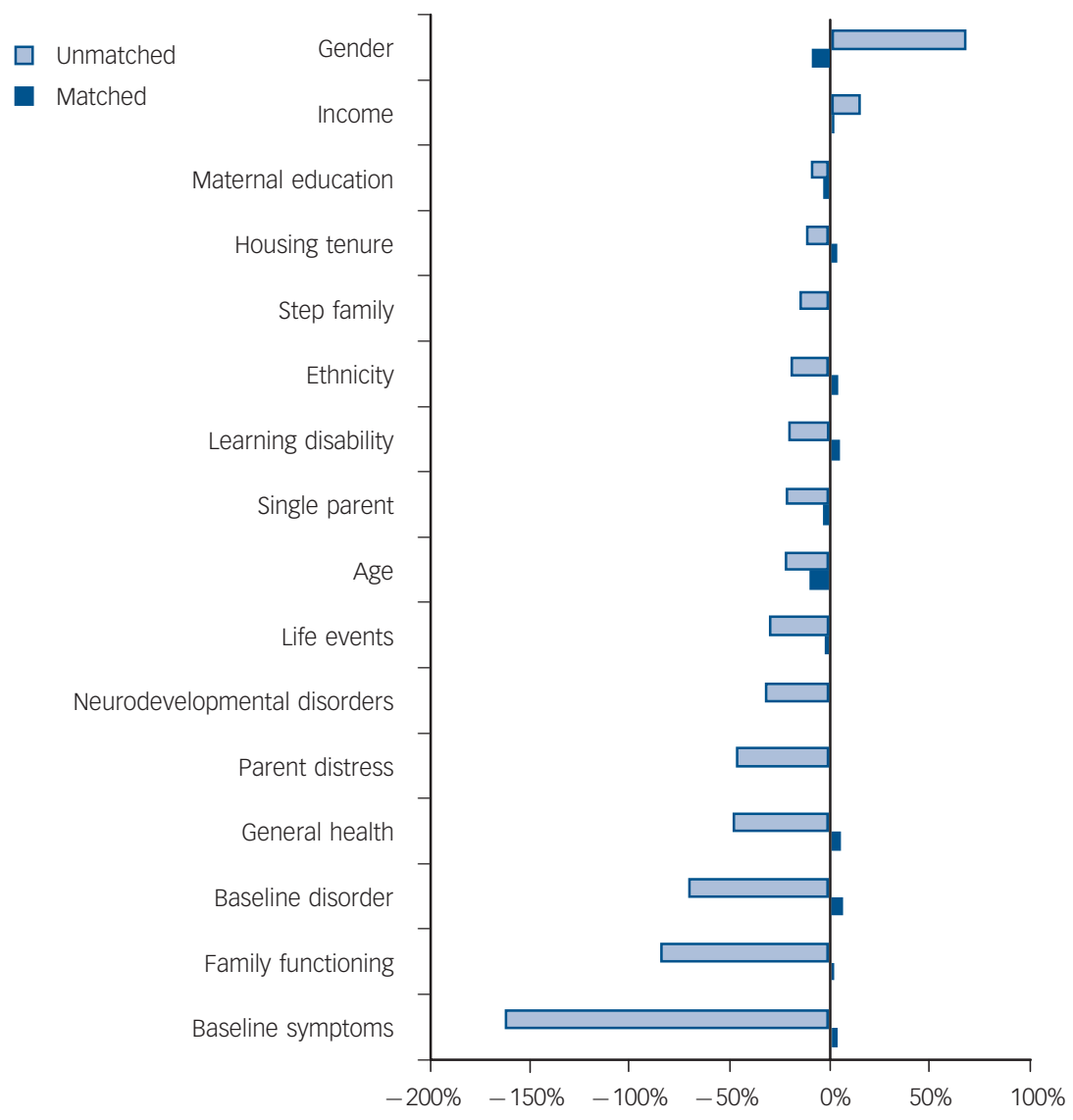

Fig. 3 Quality of propensity score matching: standardised bias (\%) across covariates before and after matching groups with high and low positive attributes. 


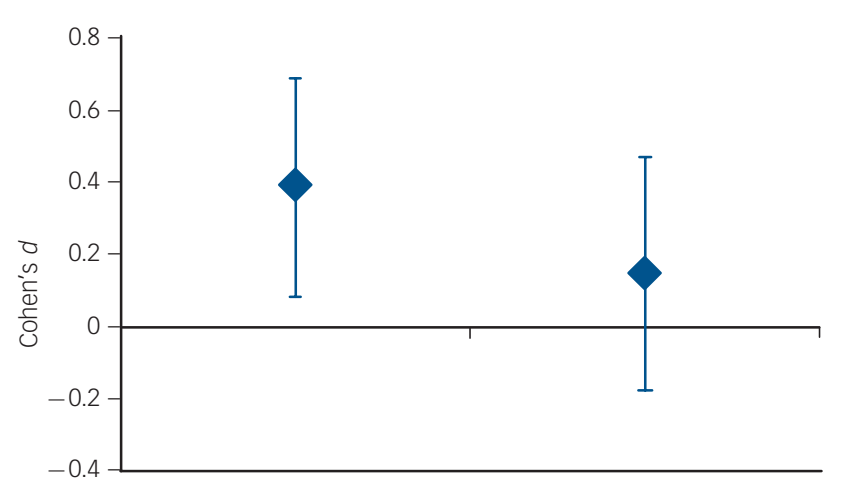

Low positive attributes

High positive attributes

Fig. 4 Standardised increase of psychopathology over time in matched groups: effect sizes of change in the Strengths and Difficulties Questionnaire total score (symptoms) at follow-up in matched groups with high and low levels of positive attributes.

psychiatric symptoms and disorders when adjusting for relevant confounders. Although effect sizes were relatively small, especially for psychiatric symptoms, predictions were particularly strong for 'all types of disorder combined' and 'externalising disorder'. The results for the self-reported positive attributes were in the line with parent-reported measures; that is, higher levels of self-reported positive attributes at baseline were significant predictors of less psychiatric symptoms at follow-up. In addition, higher levels of self-reported positive attributes were significant predictors of fewer externalising disorders. These results are available in the online Tables DS1-3.

To date, most of the literature on this topic has been based on clinical samples and has used self-reported positive attributes. To our knowledge there are very few works employing parent-report measures of positive attributes ${ }^{5,8}$ and even fewer examining the relationship with psychiatric outcomes. ${ }^{1,2}$ Using two informants, we found a moderately strong agreement between youth and parent-reported positive attributes at baseline and follow-up. We also found that levels of positive attributes were highly stable across time within parents, and moderately stable within youth and across informants.

In line with previous studies, ${ }^{2,3,37}$ higher levels of positive attributes at baseline were predictive of fewer psychiatric symptoms 3 years later. As expected, the predictions were still significant even after accounting for symptoms at baseline and relevant child and family factors. As we predicted, effect sizes were moderate in the unadjusted models (for example -0.48 for parent-reported positive attributes to symptoms 3 year later) and substantially attenuated in adjusted models (for example about -0.10 for parent-reported positive attributes to symptoms 3 years later). However, the propensity score matching approach demonstrated that children who only differ in levels of positive attributes at baseline showed different levels of psychiatric symptoms at follow-up. As expected, differences in psychiatric symptoms were lower but still significant. This approach, which is more similar to an RCT, confirms that positive attributes are independently related to psychiatric symptoms.

In addition, a path analytical model showed that positive attributes were best predicted by positive attributes and symptoms were best predicted by symptoms, suggesting different constructs. Our data are more in keeping with a vulnerability model than a model in which positive attributes and psychopathology are on the same continuum. ${ }^{38}$
Supporting previous results in adolescent samples, ${ }^{1}$ we also found that higher positive attributes scores at baseline added to the prediction of reduced risk for psychiatric disorders 3 years later. For example, our results showed that every decrease in parent-reported positive attributes score increased the probability of future psychiatric disorder (all sorts combined) by $76 \%$, of emotional disorder by $45 \%$ and of externalising disorder by $85 \%$. These probabilities were only attenuated by $10 \%$ after accounting for child and family factors. Of note, higher positive attributes scores were more likely to predict reduced risk for externalising disorders than for emotional disorders. This was an unexpected finding and should be confirmed in future studies. Previous research found a significant association with emotional disorders and any psychiatric disorder, although only the last remained significant after controlling for socioeconomic confounders. ${ }^{1}$ In contrast to our results, the same study did not find an association with disruptive disorders.

The difference in the prediction of internalising and externalising disorders might be for different reasons. First, the prevalence of each domain of disorder differs across different ages. Second, externalising problems may cause more burden in families $^{39}$ and therefore parents may underreport their children's positive attributes; however, in our sample, adjusting for parental burden did not change the pattern of the results.

In path analyses we showed that higher positive attributes at baseline predicted fewer psychiatric disorders 3 years later; this direction of effect was significantly stronger than that of psychiatric disorders predicting fewer positive attributes.

The weaker prediction to symptoms than to disorders may be explained by the methods employed for collecting the data. Psychiatric diagnoses were based on clinical ratings of interviews, including extensive open-ended comments - a substantially different method than the respondent-based YSI measure of positive attributes. By contrast, SDQ-based symptoms and YSIbased positive attributes shared a respondent-based approach, so that adjusting for baseline symptoms will substantially attenuate prediction as a result of common method variance. ${ }^{40}$ Indeed, if adjustment is restricted to child and family factors, without adjustment for baseline symptoms, effect sizes remained around -0.40 for parent-reported positive attributes predicting symptoms 3 years later.

Finally, lower parent-reported positive attributes scores increased the probability of new future contact with psychiatric services by $20 \%$ and contact with police by $33 \%$ after adjusting for baseline symptoms. Interestingly, contact with police was the category most strongly associated with externalising disorder whereas contact with psychiatric services was the category most strongly associated with all types of disorder combined.

\section{Implications}

Our findings suggest benefits of measuring positive attributes alongside symptoms in day-to-day mental health assessments of children and young people. This adds to the prediction of psychiatric symptoms and psychiatric disorders, in line with previous results. ${ }^{1,3}$ This enables clinicians to recognise a particularly high-risk group independently of the level of symptoms at admission - something that may be especially useful as regards externalising disorders. It is plausible that interventions aiming to increase the recognition of and further develop positive attributes may alleviate or prevent externalising disorders.

Furthermore, previous research suggests that strength-based assessment approaches promote family involvement in treatments ${ }^{41}$ and result in more caregiver satisfaction and treatment adherence. ${ }^{42}$ Assessing a child's positive attributes can be a less stigmatising way 
of discussing treatment needs and may make it easier for clinicians to engage families.

\section{Limitations}

The findings of this study must be considered in the light of its limitations. First, as is often the case in longitudinal studies, there was considerable attrition. Although this would be most likely to bias prevalence estimates rather than the associations between disorders and other factors, ${ }^{43}$ it has meant that those with high levels of psychopathology at baseline were less likely to participate in the study. Second, propensity score matching in nonexperimental studies cannot adjust for unobserved covariates, so residual confounding is still possible. ${ }^{12} \mathrm{~A}$ third limitation is that the YSI, unlike other longer strengths measures such as the Values in Action Inventory of Strengths (VIA-IS) ${ }^{4}$ or the Behavioral and Emotional Rating Scale (BERS) ${ }^{44}$ measures positive attributes as a single factor. Although simple and quick, this could be a disadvantage if different positive attributes show different associations with specific psychiatric outcomes. Future studies should compare the YSI with longer multiscale measures to establish what, if anything, is lost through using a brief unidimensional measure of positive attributes. Finally, it is conceptually unclear what is actually relevant in the assessment of positive attributes. That is, we cannot determine how far reported positive attributes reflect the presence of objective strengths in the child - as opposed to subjective rater biases. However, the same limitation applies to all parent-reported psychopathology, and is not specific to positive attributes. Future research to examine this issue might use a variety of informants (including teachers and peers) and approaches (including systematic observations schedules, intervention studies and qualitative studies)

To sum up, our findings suggest that positive attributes can be easily and reliably measured with the YSI from the DAWBA and can provide useful information regarding psychosocial and psychiatric outcomes in children and young people.

Pablo Vidal-Ribas, MSC, Robert Goodman, PhD, FRCPsych, Argyris Stringaris, $\mathrm{MD}, \mathrm{PhD}, \mathrm{MRCPsych}$, Department of Child and Adolescent Psychiatry, King's College London, Institute of Psychiatry, London, UK

Correspondence: Pablo Vidal-Ribas, King's College London, Institute of Psychiatry, PO 85, 16 De Crespigny Park, London SE5 8AF, UK. Email: pablo.vidal-ribas_belil@kcl.ac.uk

First received 8 Jan 2014, final revision 29 Jun 2014, accepted 10 Jul 2014

\section{Funding}

P.V.-R. received support from the Alicia Koplowitz Foundation. A.S. receives funding from the Wellcome Trust and the National Institute of Health Research UK and book royalties from Cambridge University Press.

\section{References}

1 Bromley E, Johnson JG, Cohen P. Personality strengths in adolescence and decreased risk of developing mental health problems in early adulthood. Compr Psychiatry 2006; 47: 315-24.

2 Radigan M, Wang R. Relationships between youth and caregiver strengths and mental health outcomes in community based public mental health services. Community Ment Health J 2013; 49: 499-506.

3 Gillham J, Adams-Deutsch Z, Werner J, Reivich K, Coulter-Heindl V, Linkins M, et al. Character strengths predict subjective well-being during adolescence. J Posit Psychol 2011; 6: 31-44.

4 Park N, Peterson C. Moral competence and character strengths among adolescents: the development and validation of the Values in Action Inventory of Strengths for Youth. J Adolesc 2006; 29: 891-909.
5 Park N, Peterson C. Character strengths and happiness among young children: content analysis of parental descriptions. J Happiness Stud 2006; 7 323-41.

6 Green H. Mental Health of Children and Young People in Great Britain, 2004. Palgrave Macmillan, 2005.

7 Parry-Langdon, N. (2008) Three Years On: Survey of the Emotional Development and Well-Being of Children and Young People. Office for National Statistics, 2008.

8 Williams S, McGee R. Adolescents' self-perceptions of their strengths. J Youth Adolesc 1991; 20: 325-37

9 Ford T, Goodman R, Meltzer, H. The British child and adolescent mental health survey 1999: the prevalence of DSM-IV disorders. J Am Acad Child Adolesc Psychiatry 2003; 42: 1203-11.

10 Goodman R, Ford T, Richards H, Gatward R, Meltzer H. The Development and Well-Being Assessment: description and initial validation of an integrated assessment of child and adolescent psychopathology. J Child Psychol Psychiatry 2000; 41: 645-55.

11 Austin PC. An introduction to propensity score methods for reducing the effects of confounding in observational studies. Multivariate Behav Res 2011; 46, 399-424.

12 Stuart EA Matching methods for causal inference: a review and a look forward. Stat Sci 2010; 25: 1

13 Rosenbaum PR, Rubin DB. Constructing a control group using multivariate matched sampling methods that incorporate the propensity score. Am Stat 1985; 39: 33-8

14 American Psychiatric Association. Diagnostic and Statistical Manual of Mental Disorders (4th edn, revised) (DSM-IV-TR). APA, 2000

15 World Health Organization. ICD-10: Classification of Mental and Behavioural Disorders. WHO, 1994.

16 Landis JR, Koch GG. The measurement of observer agreement for categorical data. Biometrics 1977; 33: 159-74.

17 Peterson C, Seligman MEP. Character Strengths and Virtues: A Handbook and Classification. American Psychological Association, 2004.

18 Epstein MH, Ryser G, Pearson N. Standardization of the Behavioral and Emotional Rating Scale: factor structure, reliability, and criterion validity. J Behav Health Serv Res 2002; 29: 208-16.

19 Stringaris A, Goodman R. The value of measuring impact alongside symptoms in children and adolescents: a longitudinal assessment in a community sample. J Abnorm Child Psychol 2013; 41: 1109-20.

20 Goodman R. The Strengths and Difficulties Questionnaire: a research note. J Child Psychol Psychiatry 1997; 38: 581-6.

21 Goodman, R. Psychometric properties of the strengths and difficulties questionnaire. J Am Acad Child Adolesc Psychiatry 2001; 40: 1337-45.

22 Plomin R, Haworth CM, Davis OS. Common disorders are quantitative traits. Nat Rev Genet 2009; 10: 872-8.

23 Goldberg DP, Gater R, Sartorius N, Ustun T, Piccinelli M, Gureje O, et al. The validity of two versions of the GHQ in the WHO study of mental illness in general health care. Psychol Med 1997; 27: 191-7.

24 Miller IW, Epstein NB, Bishop DS, Keitner GI. The McMaster Family Assessment Device: reliability and validity. J Marital Fam Ther 1985; 11: 345-56.

25 Goodyer I, Wright C, Altham P. The friendships and recent life events of anxious and depressed school-age children. Br J Psychiatry 1990; 156; 689-98.

26 Ford T, Collishaw S, Meltzer H, Goodman R. A prospective study of childhood psychopathology: independent predictors of change over three years. Soc Psychiatry Psychiatr Epidemiol 2007; 42: 953-61.

27 Ford T, Goodman R, Meltzer H. (2004) The relative importance of child, family, school and neighbourhood correlates of childhood psychiatric disorder. Soc Psychiatry Psychiatr Epidemiol 2004; 39: 487-96.

28 Walrath CM, Mandell DS, Holden EW, Santiago RL. Assessing the strengths of children referred for community-based mental health services. Ment Health Serv Res 2004; 6: 1-8.

29 Zanutto EL. A comparison of propensity score and linear regression analysis of complex survey data. J Data Sci 2006; 4: 67-91.

30 Dehejia RH, Wahba S. Causal effects in nonexperimental studies: reevaluating the evaluation of training programs. J Am Stat Assoc 1999; 94: 1053-62.

31 Rubin DB. The use of matched sampling and regression adjustment to remove bias in observational studies. Biometrics 1973; 29: 185-203.

32 Rubin DB. Using multivariate matched sampling and regression adjustment to control bias in observational studies. J Am Stat Assoc 1979; 74: 318-28.

33 Rubin DB, Thomas N. Combining propensity score matching with additional adjustments for prognostic covariates. J Am Stat AssoC 2000; 95: 573-85. 
34 Heckman JJ, Ichimura H, Todd P. Matching as an econometric evaluation estimator. Rev Econ Stud 1998; 65: 261-94.

35 Normand SLT, Landrum MB, Guadagnoli E, Ayanian JZ, Ryan TJ, Cleary PD, et al. Validating recommendations for coronary angiography following acute myocardial infarction in the elderly: a matched analysis using propensity scores. J Clin Epidemiol 2001; 54: 387-98.

36 Muthen LK, Muthen BO. Mplus User's Guide (7th edn). Muthén \& Muthén, 2012.

37 Lyons JS, Uziel-Miller ND, Reyes F, Sokol PT (2000) Strengths of children and adolescents in residential settings: prevalence and associations with psychopathology and discharge placement. J Am Acad Child Adolesc Psychiatry 2000; 39: 176-81.

38 Tackett JL. Evaluating models of the personality-psychopathology relationship in children and adolescents. Clin Psychol Rev 2006; 26: 584-99.
39 Meltzer H, Ford T, Goodman R, Vostanis P. The burden of caring for children with emotional or conduct disorders. Int J Family Med 2011; 2011: 801203.

40 Podsakoff PM, MacKenzie SB, Lee JY, Podsakoff NP. Common method biases in behavioral research: a critical review of the literature and recommended remedies. J Appl Psychol 2003; 88: 879-903.

41 Rudolph SM, Epstein MH. Empowering children and families through strength-based assessment. Reclaiming Children Youth 2000; 8: 207-9.

42 Cox KF. Investigating the impact of strength-based assessment on youth with emotional or behavioral disorders. J Child Fam Stud 2006; 15: 278-92.

43 Wolke D, Waylen A, Samara M, Steer C, Goodman R, Ford T, et al. Selective drop-out in longitudinal studies and non-biased prediction of behaviour disorders. Br J Psychiatry 2009; 195: 249-56.

44 Epstein MH, Sharma JM. Behavioral and Emotional Rating Scale. Pro-Ed, 1998.



psychiatry in the movies

\section{Watchmen's Rorschach}

\section{Joseph Kane}

Set in an alternate history, Watchmen tells the story of a group of retired crime fighters rendered redundant by a society in the midst of an ever-escalating Cold War. Adapted for the screen in 2009, it is arguably the most celebrated graphic novel of all time.

Watchmen's chief protagonist is Walter Kovacs, the son of a cold, abusive mother, who did little to shield young Walter from her life as a prostitute. Author Alan Moore interweaves fact and fiction as the 1964 murder of New Yorker Kitty Genovese prompts Kovacs, disgusted at the apathy of the 38 witnesses that reportedly failed to intervene in the crime, to adopt his alter ego, Rorschach. Named after German psychoanalyst Hermann Rorschach, Watchmen's antihero dons a distinctive mask that features moving, symmetrical patterns resembling the famous inkblot tests created by his namesake, reflecting his black and white view of a society that repulses him.

Consistent with the archetype of the masked vigilante, the character is reminiscent of, among others, Batman, Don Quixote, and Charles Bronson's portrayal of Paul Kersey in the Death Wish series of films. Scarred by his past and motivated by a tenacious dedication to his moral crusade, Rorschach exacts violent retribution on the criminals he perceives as animals: 'Men get arrested. Dogs get put down'.

When Edward 'The Comedian' Blake is murdered, the other members of the Watchmen, many of whom have abandoned their crime-fighting personas, dismiss Rorschach's hypothesis that a 'mask killer', hell-bent on assassinating former heroes, is at large. Viewed by his former colleagues as a loose cannon, paranoid and out of touch, Rorschach's characteristic implacable pursuit of the case uncovers disturbing evidence that an even greater conspiracy exists.

Rorschach's tense consultations with forensic psychiatrist Dr Malcolm Long, shortly after he is framed and imprisoned for the murder of a former criminal kingpin, give the reader their first glimpse of the man behind the mask. Long, although well-meaning and adamant that he can rehabilitate his patient, is greatly disturbed by Kovacs' justification for his actions and beliefs, eventually leading him to question his own principles and wreaking havoc on his private life.

The story climaxes in the Watchmen's confrontation of the mask killer while the USA and the USSR teeter on the brink of nuclear war. As both the reader and each of the main characters are confronted with a challenging, thought-provoking ultimatum, Rorschach maintains his unwavering philosophy to the very last: 'Never compromise. Not even in the face of Armageddon'. 